\title{
Frequency-dependent Seismic Anisotropy of Porous Rocks with Penny-shaped Cracks
}

\author{
Luke Brown' Boris Gurevich ${ }^{2}$
}

Key Words: poroelasticity, fractures, anisotropy, dispersion

\begin{abstract}
Porous reservoirs with aligned fractures exhibit frequencydependent seismic anisotropy because of wave-induced fluid flow between pores and fractures. To relate the elastic properties of porous rocks with aligned fractures at low frequency, we use the linear slip model of fractures and anisotropic Gassmann fluid substitution. We combine this low-frequency anisotropic Gassmann model with a dispersion relationship, based on a pennyshaped crack model of fractures, to account for frequencydependent anisotropy.
\end{abstract}

The combined model is validated using experimental measurements of angle-dependent wave velocities of synthetic porous sandstone with aligned disc-shaped cracks. For the lowfrequency anisotropic Gassmann model, the agreement between the measured and predicted velocities is reasonably good for both $S$-wave velocities, but $\mathrm{P}$-wave anisotropy is overestimated by approximately $25 \%$. This quantitative difference can be explained by fluid diffusion effects occurring at the relatively high frequencies used in the experiment $(100 \mathrm{kHz})$, which are not accounted for by the low-frequency assumption of anisotropic Gassmann theory. The predictions of the combined frequencydependent model, which considers this effect, give very good agreement with measured velocities.

\section{INTRODUCTION}

Natural fractures often control permeability and fluid flow, and hence the distribution and recovery of reserves, in fractured reservoirs. The elastic properties and seismic characteristics of fluid-saturated porous rocks with aligned fractures may be used to locate fractured areas with high permeability in a reservoir. Such reservoir characterisation requires expressions relating the properties of the fractures and pore fluid to the elastic properties of the fractured reservoir rock.

Department of Exploration Geophysics

Curtin University of Technology

GPO Box U1987

Western Australia, Australia 6845

Phone: (08) 92663519

Fax: (08) 92663407

Email: luke.brown@geophy.curtin.edu.au

2 Department of Exploration Geophysics

Curtin University of Technology

GPO Box $U 1987$

Western Australia, Australia 6845

Phone: (08) 92667359

Fax: (08) 92663407

Email: boris.gurevich@geophy.curtin.edu.au

Presented at the 16th ASEG Geophysical Conference \& Exhibition February 2003.

Revised manuscript received: 23 March, 2004
The effect of fractures on the elastic properties of fluid saturated porous reservoirs differs from their effect in non-porous elastic media through two mechanisms: wave-induced fluid flow between the pores and fractures, and seismic scattering. Porous rocks with aligned fractures therefore exhibit frequency-dependent anisotropy, and hence require expressions for the elastic properties as functions of frequency.

At low frequencies, the elastic properties of a saturated isotropic (non-fractured) porous rock are commonly determined using the isotropic Gassmann equations. However, rocks with aligned fractures are anisotropic, and thus require a fluid substitution model that accounts for anisotropy. To model the elastic properties of porous media with aligned fractures, Gurevich (2002) proposed a combination of the linear slip interface model of fractures (Schoenberg and Douma, 1988; Schoenberg and Sayers, 1995) combined with anisotropic Gassmann (1951) equations.

Similarly to the isotropic Gassmann equations, this approach assumes pressure equilibration between pores and fractures, and thus is only valid in the low-frequency limit. A model for frequency-dependent elastic properties of porous rocks with aligned cracks was proposed by Hudson et al. (1996, 2001). However, since this model is based on the analysis of wave interaction with a single crack, its low-frequency predictions are not consistent with anisotropic Gassmann theory.

To model frequency-dependent anisotropy in saturated porous rocks with aligned fractures, we combine the anisotropic Gassmann model (Gurevich, 2002) with the dispersion relationship of Hudson et al. (1996). We analyse the validity of both the low-frequency model and the combined frequencydependent model by comparing predicted velocities to measured velocities from the ultrasonic experiment of Rathore et al. (1995).

\section{THEORY}

\section{Low-frequency theory}

The model proposed by Gurevich (2002) assumes that the dry rock is a spatially homogeneous and isotropic porous rock (host rock) permeated by a set of parallel fractures. The host rock is assumed to be made up of a single isotropic elastic grain material with bulk modulus $K_{g}$. The host rock is characterized by porosity $\phi$ and Lamé constants $\lambda$ and $\mu$. The host rock is permeated by a set of parallel fractures, which are defined using the linear slip model (Schoenberg and Douma, 1988). According to this model, an elastic medium permeated by a single set of parallel fractures can be characterized by the compliance matrix

$$
\mathbf{s}^{0}=\mathbf{s}_{b}+\mathbf{s}_{e},
$$

where $\mathbf{s}_{b}$ is the compliance matrix (inverse of stiffness matrix) of the host rock and $\mathbf{s}_{e}$ is the excess compliance matrix associated with the fractures, which for rotationally invariant fractures parallel to $y-z$ plane is 


$$
\mathbf{s}_{e}=\left(\begin{array}{cccccc}
Z_{N} & 0 & 0 & 0 & 0 & 0 \\
0 & 0 & 0 & 0 & 0 & 0 \\
0 & 0 & 0 & 0 & 0 & 0 \\
0 & 0 & 0 & 0 & 0 & 0 \\
0 & 0 & 0 & 0 & Z_{T} & 0 \\
0 & 0 & 0 & 0 & 0 & Z_{T}
\end{array}\right)
$$

The non-zero elements $Z_{N}$ and $Z_{\tau}$ of the matrix $\mathbf{s}_{c}$ are the normal and shear excess compliances that relate the displacements and stresses applied to the fractures in the normal and tangential directions. An isotropic medium with rotationally invariant fractures is transversely isotropic (TI), with a symmetry axis perpendicular to the fracture plane. General TI media are defined by five independent elastic parameters $\left(c_{11}, c_{33}, c_{13}, c_{44}\right.$, and $c_{55}=$ $c_{66}$ ). In the stiffness domain, equation (1) yields the following expressions for the five independent elements of the stiffness matrix $\mathbf{c}^{0}$ :

$$
\begin{aligned}
& c_{11}^{0}=L\left(1-\Delta_{N}\right), \quad c_{33}^{0}=L\left(1-\frac{\lambda^{2}}{L^{2}} \Delta_{N}\right), \\
& c_{13}^{0}=\lambda\left(1-\Delta_{N}\right), \quad c_{44}^{0}=\mu, \\
& \text { and } \quad c_{55}^{0}=\mu\left(1-\Delta_{T}\right),
\end{aligned}
$$

where $L=\lambda+2 \mu$, while $\Delta_{N}$ and $\Delta_{T}$ are dimensionless fracture weaknesses given by

$$
\Delta_{N}=\frac{(\lambda+2 \mu)}{1+(\lambda+2 \mu) Z_{N}}, \text { and } \Delta_{T}=\frac{\mu Z_{T}}{1+\mu Z_{T}}
$$

(Schoenberg and Douma, 1988).

The isotropic Gassmann equations, commonly used to determine the elastic properties of isotropic porous medium, do not account for anisotropy and therefore cannot be applied to fractured media. However, Gassmann (1951) presented similar equations for an anisotropic medium composed of a single isotropic grain material, which allow us to determine the effect of a saturating fluid on the elastic properties of fractured porous rock. These equations were later generalised by Brown and Korringa (1975) for rocks with heterogeneous grain material.

When stiffness components inverted from the dry rock compliance matrix are substituted into the anisotropic Gassmann equations, they yield expressions for the stiffness of the saturated rock in terms of host rock moduli, fracture weaknesses, and fluid bulk modulus $K_{f}$ (Gurevich, 2002)

$$
\begin{gathered}
c_{11}^{s a t}=\frac{L}{D}\left[d_{1} \theta+\frac{K_{f}}{\phi K_{g} L}\left(L_{1} \alpha^{\prime}-\frac{16}{9} \frac{\mu^{2} \alpha_{0}}{L} \Delta_{N}\right)\right], \\
c_{33}^{\text {sat }}=\frac{L}{D}\left[d_{2} \theta+\frac{K_{f}}{\phi K_{g} L}\left(L_{1} \alpha^{\prime}-\frac{4}{9} \frac{\mu^{2} \alpha_{0}}{L} \Delta_{N}\right)\right], \\
c_{13}^{s a u t}=\frac{\lambda}{D}\left[d_{1} \theta+\frac{K_{f}}{\phi K_{g} \lambda}\left(\lambda_{1} \alpha^{\prime}-\frac{8}{9} \frac{\mu^{2} \alpha_{0}}{L} \Delta_{N}\right)\right], \\
c_{44}^{s a t}=\mu, \text { and } \quad c_{55}^{s a t}=\mu\left(1-\Delta_{T}\right),
\end{gathered}
$$

where the following notation has been used for compactness,

$$
\begin{aligned}
& K=\lambda+\frac{2 \mu}{3}, \\
& L=\lambda+2 \mu, \\
& D=1+\frac{K_{f}}{K_{g} \phi}\left(\alpha_{0}-\phi+\frac{K^{2} \Delta_{N}}{K_{g} L}\right), \\
& \theta=1-\frac{K_{f}}{K_{g}} \\
& \alpha^{\prime}=\alpha_{0}+\frac{K^{2}}{K_{g} L} \Delta_{N}, \\
& L_{1}=K_{g}+\frac{4}{3} \mu \\
& \lambda_{1}=K_{g}-\frac{2}{3} \mu, \\
& d_{1}=1-\Delta_{N}, \text { and } \\
& d_{2}=1-\frac{\lambda^{2}}{L^{2}} \Delta_{N} .
\end{aligned}
$$

Equations (5)-(8) provide explicit expressions for the elastic properties of a saturated porous rock with aligned fractures.

The degree of anisotropy of a TI medium is defined by Thomsen's (1986) anisotropy parameters $\varepsilon, \delta$, and $\gamma$, which describe the variation of $\mathrm{P}$ - and $\mathrm{S}$-wave velocities as a function of angle to the symmetry axis:

$$
\varepsilon=\frac{c_{33}-c_{11}}{2 c_{11}}, \gamma=\frac{c_{44}-c_{55}}{2 c_{55}}, \text { and } \delta=\frac{\left(c_{13}+c_{55}\right)^{2}-\left(c_{11}-c_{55}\right)^{2}}{2 c_{11}\left(c_{11}-c_{55}\right)}
$$

The anisotropy parameters are determined by substituting the fluid saturated rock stiffness components from equations (5)-(8) into equations (9).

\section{FREQUENCY-DEPENDENT THEORY}

Anisotropic Gassmann theory assumes fluid pressure equilibrium between the pores and fractures, and is therefore only valid in the low-frequency limit. To satisfy this condition, the fluid diffusion length $J$

$$
J=\sqrt{\phi K_{f} \kappa / 2 \eta \omega}
$$

must be much larger than fracture size and spacing (Hudson et al., 2001). In equation (10), $\omega$ is frequency, $\kappa$ is permeability of the host rock, and $\eta$ is dynamic fluid viscosity. At higher frequencies, when fracture size is larger than fluid diffusion length, the fluid does not have sufficient time to flow between the pores and fractures.

A model for frequency-dependent elastic properties of porous rocks with aligned penny-shaped cracks was proposed by Hudson et al. (1996), based on the analysis of fluid pressure relaxation between a single penny-shaped crack and the porous host rock. According to this model, for a medium with aligned penny-shaped cracks with crack density $e$, thickness $c$ and diameter $a$, Thomsen's (1986) anisotropy parameters can be rewritten as 


$$
\begin{aligned}
& \varepsilon^{s a t}=\frac{8 e}{3}(1+F), \\
& \gamma^{\text {sat }}=\frac{8 e}{3}\left(\frac{1-v^{s a t}}{2-v^{s a t}}\right), \text { and } \\
& \delta^{\text {sat }}=2\left(1-2 v^{\text {sat }}\right) \varepsilon^{s a t}-2\left(\frac{1-2 v^{\text {sat }}}{1-v^{\text {sat }}}\right) .
\end{aligned}
$$

In equation (11), vat is Poisson's ratio of the saturated host rock and $F$ is a frequency factor defined as

$$
F=\frac{1}{\pi} \frac{a}{c}\left(\frac{\lambda^{s a t}+2 \mu}{\lambda^{s a t}+\mu}\right) \frac{K_{f}}{\mu}[1+3(1-i) J / c]^{-1}
$$

where $\lambda^{\text {sat }}$ is the Lamé coefficient of the saturated host rock. As shown in equation (10), for the case of infinitely low frequencies $\omega \rightarrow 0$, diffusion length is much larger than fracture size $J>a \gg c$. Substituting this result into equation (12) gives a small frequency factor $F \ll 1$, and hence according to equation (11), the anisotropy parameter $\varepsilon$ of the saturated medium is $\varepsilon \approx \frac{8}{3} e$. Therefore, in the low-frequency limit, the predicted anisotropy parameter $\varepsilon$ of the saturated medium is equivalent to that of the dry medium (see equations (11) and (12) with $K_{f}=0$ ). Therefore, as explained by Hudson et al. (2001), the equant porosity model of Hudson et al. (1996) is not consistent with anisotropic Gassmann theory (Gassmann, 1951; Brown and Korringa, 1975), and is not strictly valid in the low-frequency limit.

To model frequency-dependent anisotropy in fractured porous rocks over a broad frequency range, we combine the dispersion relationship of Hudson et al. (1996) with the low-frequency anisotropic Gassmann model of Gurevich (2002). The frequency factor $F$ given in equation (12) is used to scale the stiffness coefficients between their minimal and maximal values in the low and high frequency limits respectively.

$$
c_{i j}(\omega)=c_{i j}^{0}+\left(c_{i j}^{m}-c_{i j}^{0}\right)\left(1-\frac{1}{1+F}\right)
$$

At low frequencies $F=0$, and the low-frequency stiffness coefficients $c_{i j}^{0}$ are given by anisotropic Gassmann theory according to equations (5) to (8). At high frequencies, the lowfrequency terms cancel, and the high-frequency stiffness coefficients $c_{i j}^{\infty}$ are given by well-known theory for isolated cracks in elastic media. To validate this combined frequency-dependent model, we compare its predictions to results from the experiment of Rathore et al. (1995).

\section{EXPERIMENTAL VALIDATION}

\section{Description of the experiment}

Rathore et al. (1995) conducted an experiment allowing effectivemedium theories to be tested on a synthetic porous rock with cracks of known geometry. Synthetic sandstone was constructed using sand grains cemented with an epoxy resin, and thin metal discs of known size, shape, orientation and distribution were emplaced during construction. The metal discs were later extracted by chemical leaching, resulting in residual disc-shaped cracks in a consolidated porous rock. Two synthetic rock samples, dummy sample 901D with no cracks and sample $901 \mathrm{C}$ with cracks, were manufactured to determine anisotropy associated with the fractures.

The sandstone host rock had a porosity of 0.346 and a density of $1712 \mathrm{~kg} \mathrm{~m}^{-3}$. The circular cracks were $0.02 \mathrm{~mm}$ thick and $5.5 \mathrm{~mm}$ in diameter (aspect ratio of 0.0036 ), with a crack density of 0.10 and crack porosity of 0.0023 .
The velocities of the quasi P-wave $\left(V_{P}\right)$, vertically polarised quasi $\mathrm{S}$-wave $\left(V_{s v}\right)$, and the horizontally polarised $\mathrm{S}$-wave $\left(V_{S H}\right)$ were measured at different incident angles to the symmetry axis of the cracks. The dummy (uncracked) sample showed uniform arrival times for all angles, while the cracked sample showed variation of arrival times with angle because of crack induced anisotropy (Rathore et al., 1995). The P-wave anisotropy, defined by the difference between the maximum and minimum $\mathrm{P}$-wave velocity, was much larger in the dry cracked sample than in the saturated cracked sample.

\section{Previous studies}

Rathore et al. (1995), Thomsen (1995) and Hudson et al. (2001) fitted velocities measured in the direction parallel to the fractures and used the penny-shaped crack model of fractures to predict angle-dependent velocities. This method independently predicted the elastic properties of the dry and saturated samples by fitting the corresponding velocities measured on the dry and saturated samples. The elastic properties of the saturated sample were not directly related to the properties of the dry sample during these studies.

The anisotropic Gassmann model predicts the elastic properties of a saturated porous rock with aligned fractures from the independent properties of the host rock, fractures and fluid. To relate the properties of the saturated rock to the properties of the dry rock, we use an alternative method that does not require any fitting to measurements made on the saturated fractured sample.

\section{Method}

The properties of the dry rock and fractures were fitted to measurements made on the dry dummy and dry cracked samples. The properties of the saturated fractured rock were then predicted from the properties of the dry rock, fractures, and fluid, using anisotropic Gassmann fluid substitution.

The anisotropic Gassmann model required the elastic moduli $K$ and $\mu$ of the dry host rock, normal and tangential fracture weaknesses $\Delta_{N}$ and $\Delta_{T}$, and bulk moduli of the solid grains $K_{\mathrm{g}}$ and fluid $K_{f}$. These input parameters, excluding fluid bulk modulus, were predicted using the following procedure.

- Dry host moduli $K$ and $\mu$ were fitted to the P-and S-wave velocities measured on the dry dummy (uncracked) sample, and density $\rho$.

- The bulk modulus of the grain material $K_{g}$ was estimated using the isotropic Gassmann equation, and P-wave velocities measured on the dry and saturated dummy sample. The predicted bulk modulus of $30 \mathrm{GPa}$ is reasonable for quartz sandstone with $4 \%$ added epoxy.

- Normal $\Delta_{N}$ and tangential $\Delta_{T}$ fracture weaknesses were estimated by fitting dry $\mathrm{P}$ and $\mathrm{S}$ velocities predicted from equations (3) to the velocities measured on the dry fractured sample in the symmetry directions $\left(0^{\circ}\right.$ and $\left.90^{\circ}\right)$ (Figure 1)

The fit between the measured and fitted velocities, as shown in Figure 1, is not very good at intermediate angles away from the symmetry directions. While we model only phase velocities, the measurements represent a mixture of phase and group velocities of mixed modes in an anisotropic medium. The misfit is relatively large in this case because the anisotropy is stronger in dry fractured rocks. Since phase and group velocities coincide in symmetry directions, we fitted to velocities measured in these directions, and allowed the discrepancy at other angles. 


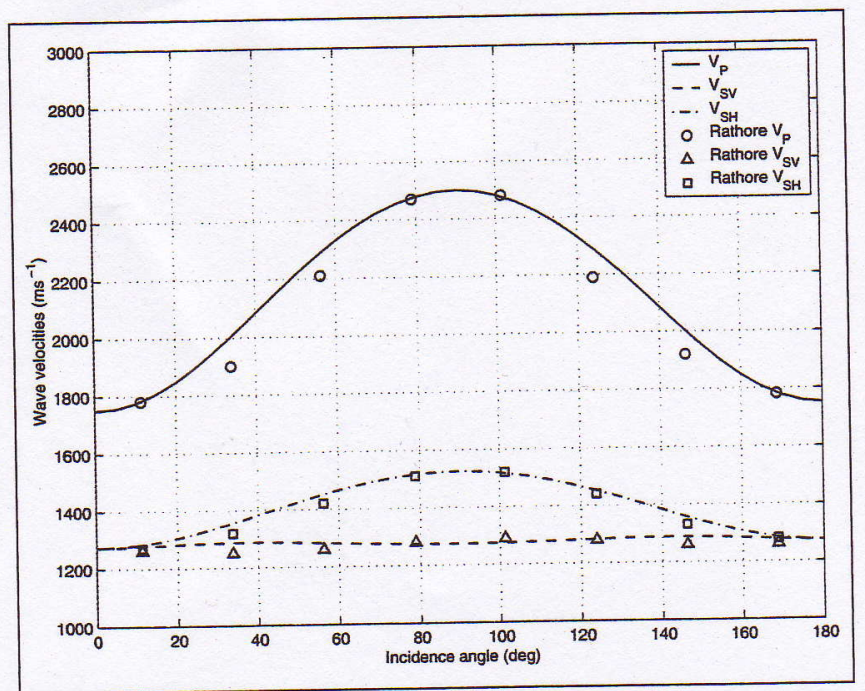

Fig. 1. Fitting of measured (symbols) and predicted (lines) velocities for dry fractured sample using linear slip model.

The parameters above define the stiffness matrix describing the elastic properties of the dry fractured rock. The angle-dependent Pand S-wave velocities were predicted using components of the dry and saturated stiffness matrices, related by (Mavko et al., 1998),

$$
\begin{gathered}
V_{P}=\left(c_{33} \sin ^{2} \theta+c_{11} \cos ^{2} \theta+c_{66}+M\right)^{1 / 2}(2 \rho)^{-1 / 2}, \\
V_{S V}=\left(c_{33} \sin ^{2} \theta+c_{11} \cos ^{2} \theta+c_{66}-M\right)^{1 / 2}(2 \rho)^{-1 / 2}, \\
V_{S H}=\left(\frac{c_{44} \sin ^{2} \theta+c_{66} \cos ^{2} \theta}{\rho}\right)^{1 / 2},
\end{gathered}
$$

where

$$
M=\left\{\left[\left(c_{33}-c_{66}\right) \sin ^{2} \theta-\left(c_{11}-c_{66}\right) \cos ^{2} \theta\right]^{2}+\left(c_{13}+c_{66}\right)^{2} \sin ^{2} 2 \theta\right\}^{1 / 2},
$$

and $\theta$ is the angle of incidence relative to the axis of symmetry.

The additional input parameter required for the frequency correction was the permeability of the samples, not supplied by Rathore et al. (1995). Hudson et al. (2001) predicts permeability using a least square fit, however, the permeability value obtained $(\sim 0.4 \mathrm{D})$ is relatively low considering the grain size and porosity of the samples. The actual measured permeability of the synthetic samples, as supplied by E. Fjær of IKU, was 11.4 D, substantially higher than the fitted permeability of Hudson et al. (2001).

The measured permeability of 11.4 Darcy may seem much higher than usually reported for natural rocks. However, the rock used in the experiments of Rathore et al. (1995) is not natural, but rather a synthetic sandstone composed of clean, coarse grained and well-sorted sand. Considering the properties of the sand, and the unusually high porosity of the synthetic samples $(0.35)$, the measured permeability is within the realistic range for such rocks. For example, Figure 1.9 from Bourbie et al. (1987) shows that for well-sorted, coarse grained, clean sandstones with porosity of 0.35 , permeability is in the order of $10 \mathrm{D}$.

\section{Results}

Figure 2 compares the angle dependent $\mathrm{P}$ - and $\mathrm{S}$-wave velocities predicted using the anisotropic Gassmann model, to the

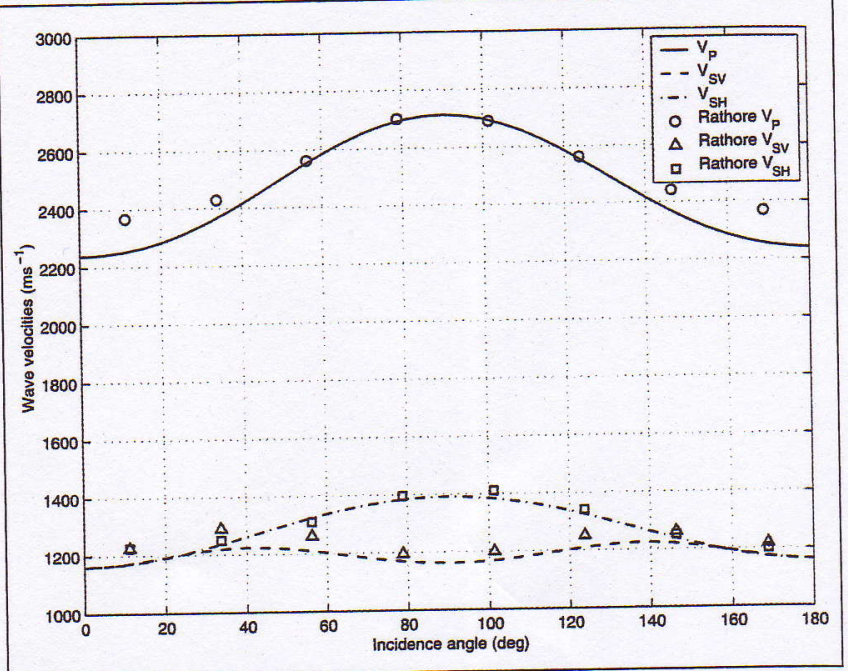

Fig. 2. Comparison of measured (symbols) and predicted velocities (lines) for saturated fractured sample using low-frequency anisotropic Gassmann model.

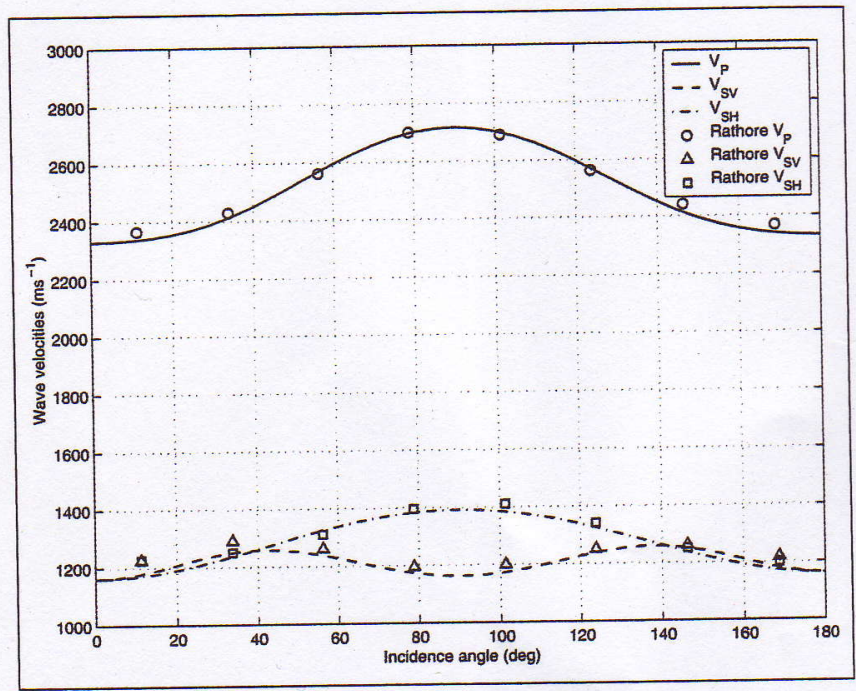

Fig. 3. Comparison of measured (symbols) and predicted velocities (lines) for saturated fractured sample using frequency-dependent model.

velocities measured on the saturated cracked sample. There is a good qualitative fit with the measured velocities, but $\mathrm{P}$-wave anisotropy is overestimated by approximately $25 \%$. The quantitative difference is thought to result from high-frequency fluid diffusion effects measured on the saturated cracked sample.

The Rathore et al. (1995) experiment was conducted at ultrasonic frequencies using a pulse with a central frequency of $100 \mathrm{kHz}$. Substituting this frequency into the expression for the diffusion length, equation (10), we obtain

$$
J=\sqrt{\frac{(0.346)\left(2.25 \times 10^{9}\right)\left(11.4 \times 10^{-12}\right)}{\left(2 \times 10^{-3}\right)\left(2 \pi \times 10^{5}\right)}}=2.6 \mathrm{~mm}
$$

For the low-frequency anisotropic Gassmann theory to be valid, this diffusion length must be much larger than the diameter of the cracks $(5.5 \mathrm{~mm})$. This is clearly not the case for the given experimental parameters, therefore a frequency-dependent model, such as the combined model we propose, is required. 
Figure 3 compares the angle-dependent P- and S-wave velocities measured on the saturated cracked sample to the velocities predicted using the combined frequency-dependent model. There is a very good quantitative fit between the predicted and measured velocities for both P-and S-waves, implying that the combined model accounts for fluid diffusion effects at relatively high frequencies.

\section{CONCLUSIONS}

In the low-frequency limit, diffusion length is large compared to fracture size and spacing, and the fluid has sufficient time to flow between the pores and fractures, equilibrating hydraulic pressure. In such a relaxed fluid flow regime, the effect of fluid saturation on the elastic properties of a porous rock with aligned fractures can be predicted using anisotropic Gassmann fluid substitution. At higher frequencies, however, fluid diffusion length decreases relative to the fracture size and spacing, and waveinduced fluid flow occurs between the pores and fractures. This wave-induced fluid flow causes frequency-dependent seismic anisotropy in fractured porous rocks, a result that is not modelled by low-frequency anisotropic Gassmann theory.

The experimental velocity measurements of Rathore et al. (1995) were made using ultrasonic frequencies much higher than allowed by the low-frequency assumption of anisotropic Gassmann theory. Therefore, because of the significant velocity dispersion in the ultrasonic measurements, the P-wave anisotropy predicted using anisotropic Gassmann theory was higher than measured in the experiment. To predict the elastic properties of a fractured porous rock as a function of frequency, a frequencydependent model of elastic properties of porous fractured rock is required. Such a model was proposed in this paper by modifying the equant porosity model of Hudson et al. (2001). The proposed model not only accounts for wave-induced fluid flow between the pores and fractures but, unlike the frequency-dependent equant porosity model, is also consistent with anisotropic Gassmann equations in the low-frequency limit. The velocities predicted using our frequency-dependent model yield very good agreement with velocities measured by Rathore et al. (1995).

\section{ACKNOWLEDGEMENTS}

The authors thank E. Fjær of IKU, Norway, for the contribution of the permeability parameter from the Rathore et al. (1995) experiment. The support of the Commonwealth Scientific Industrial Research Organisation (CSIRO), Centre of Excellence for Exploration and Production Geophysics (CEEPG), Australian Petroleum Cooperative Research Centre (APCRC), and Curtin Reservoir Geophysics Consortium (CRGC) is gratefully acknowledged.

\section{REFERENCES}

Bourbie, T., Coussy, O., Zinszner, B., 1987, Acoustics of porous media: Elf Geoscience Research Centre.

Brown, R.J.S., and Korringa, J., 1975, On the dependence of the elastic properties of a porous rock on the compressibility of the pore fluid: Geophysics, 40, 608-616.

Gassmann, F., 1951, Über die Elastizität poröser Medien: Viertel. Naturforsch. Ges. Zurich, 96, 1-23.

Gurevich, B., 2002, Elastic properties of saturated porous rocks with aligned fractures: Expanded Abstracts, 72nd Annual Meeting and Exposition, Society of Exploration Geophysicists, Paper ANI P1.6.

Hudson, J.A., Liu, E., and Crampin, S., 1996, The mechanical properties of materials with interconnected cracks and pores: Geophys. J. Internat., 124, 105-112.

Hudson, J.A., Pointer, T., and Liu, E., 2001, Effective medium theories for fluid saturated material with aligned cracks: Geophys. Prosp., 49, 509-522.

Mavko, G., Mukerii, T., and Dvorkin, J., 1998, The rock physics handbook: Cambridge University Press.

Rathore, J.S., Fjaer, E., Holt, R.M., and Renlie, L., 1995, P- and S-wave anisotropy of a synthetic sandstone with controlled crack geometry: Geophys. Prosp., 43, 711-728.

Schoenberg, M., and Douma, J., 1988, Elastic-wave propagation in media with parallel fractures and aligned cracks: Geophys. Prosp., 36, 571-590.

Schoenberg, M., and Sayers, C.M., 1995, Seismic anisotropy of fractured rock: Geophysics, 60, 204-211.

Thomsen, L., 1986, Weak elastic anisotropy: Geophysics, 51, 1954-1966.

Thomsen, L., 1995, Elastic anisotropy due to aligned cracks in porous rock: Geophys. Prosp., 43, 805-829. 